\section{Citation-based criterion for identifying long-lasting research papers}

\author{
Shubhada Nagarkar ${ }^{1, *}$ and Shridhar R. Gadre ${ }^{2}$ \\ ${ }^{1}$ Department of Library and Information Science, and \\ ${ }^{2}$ Interdisciplinary School of Scientific Computing and Department of \\ Chemistry, Savitribai Phule Pune University, Pune 411 007, India
}

This work presents the development and testing of a protocol for identifying long-lasting (L-L) research papers for which citation data of at least 25 years are available. In the first step, papers having 100 or more citations are shortlisted. The time interval from the year of publication till 2019 is divided into four approximately equal quarters. The $R_{4}$ parameter is defined as the ratio of the citations received by a paper in the most recent quarter to its total citations. Papers with $\boldsymbol{R}_{4}$ less than a prescribed cut-off value are eliminated. The trends in the citations of papers in the most recent five years are assessed next. Papers with less than a specified value of actual/extrapolated citations are omitted. For testing purpose, we have explored the citation patterns of 1,402 shortlisted papers, out of a total of 123,993 published from India between 1985 and 1994, resulting into $676 \mathrm{~L}-\mathrm{L}$ papers. We find that not all papers published in high-impact journals as well as those receiving large citations fulfil the L-L criterion. Subject-wise analysis brings out the disciplines making lasting contributions to research.

Keywords: Citation analysis, high-impact journals, impact factor, long-lasting research papers.

CITATION index was designed for effective information retrieval in which citations are the formal, explicit conceptual linkage between two research articles ${ }^{1}$. It started with the purpose of identifying original publication in which an idea/concept was discussed, and to identify many deserving but unrecognized scientists. Currently, the citation data are being used for a variety of purposes. Based on the number of citations received per paper, researchers have developed various indicators/metrics. Moreover, patterns of citations have also been studied to measure the impact of an individual research paper.

The number of citations received by a research paper has been used to build-up scores/metrics such as the impact factor (IF) of a journal, $h$-index, $g$-index, $i 10$ index for a researcher, etc. Other citation-based scores such as Citescore, Simago Journal Ranking (SJR) and Source Normalized Impact per Paper (SNIP) have also been proposed ${ }^{2}$. Comparative studies of these indicators, analysing their use and limitations for measuring the research impact have also been reported ${ }^{3-5}$.

Durability of a scientific paper is reflected in its citation history. A variety of citation patterns of research

*For correspondence. (e-mail: shubha@unipune.ac.in) papers are seen, e.g. delayed rise, medium rise, slow decline, etc. and these patterns may change according to the field of study ${ }^{6-10}$. The term 'sleeping beauty' was coined and explained by van Raan ${ }^{11}$ as an article that goes unnoticed (sleeps) for a long time and then, almost suddenly, attracts a lot of attention. The 'flash in the pan' is another pattern of citations in which a paper that is noticed immediately after publication and frequently cited, but does not seem to have a lasting impact and dies early in life ${ }^{12}$.

Walters ${ }^{13}$ stated that longitudinal studies are required for tracking the citation life cycle of scientific articles from birth. This gives an opportunity to study these papers in-depth, which is not possible in case of shortterm citation window period. Wang et al. ${ }^{14}$ discussed the pros and cons of the time period selected for citation analysis studies. They found that a short-term window period of citations may be good for the field of science and technology, but may not be suitable for disciplines such as sociology and mathematics ${ }^{14}$.

In a classic study by $\mathrm{Abt}^{15}$, the impact of long-term citations in the field of astronomy/astrophysics was measured. The citation history of 326 papers published in 1961 and cited in 18 subsequent years, encompassing 6070 citations was studied ${ }^{15}$. Results indicated that the average of 19 citations per paper or 1.0 citation per paper per year was received. These numbers would now appear to be small, the reason being that journals were in print format and pertained to pre-information explosion era. $\mathrm{Abt}^{15}$ mentions that ' 20 years after publication, the citation rate is half-maximum. It is obvious that the decline cannot be linear to the end because it would predict no citations 32 years after publication', whereas he argues that recent research papers and the Science Citation Index list many papers that are more than 32 years old. Nash et $a l .{ }^{16}$ studied the contributing factors to long-term citation count in marine and freshwater biology articles. They found that articles received early citations due to early visibility (discoverability). Kosteas ${ }^{17}$ examined the relative strength of short-term citations, IF and journal ranking in terms of predicting long-term citations. The results indicated that short-run citation counts measured through the year following publication are a better predictor of long-run citations than IF and journal ranking scores combined.

Some studies describe the relationship between longterm citations and journal IF. Stegehuis et al. ${ }^{18}$ used the journal IF and number of citations in the first year as predictors of the long-term citations. Abramo et al. ${ }^{19}$ tested the hypothesis that good predictions of long-term citations can be obtained through a combination of early citations of a paper and the journal IF. More recently, Xiao et $a l .^{20}$ proposed a model to predict the long-term impact of individual publications based on early citations. According to Leydesdorff et al. $^{21}$, short-term citations can be considered as citation currency whereas long-term 


\section{RESEARCH COMMUNICATIONS}

Table 1. Number of research papers published from India between 1985 and 1994 along with citations received up to 2019 and the number of papers receiving $\geq 100$ citations up to 2019 . See text for details

\begin{tabular}{lrcc}
\hline Year of publication & Total no. papers & Total no. of citations & No. of papers with $\geq 100$ citations \\
\hline 1985 & 9795 & 104,899 & 77 \\
1986 & 9641 & 102,738 & 84 \\
1987 & 10,329 & 111,636 & 94 \\
1988 & 11,680 & 120,583 & 110 \\
1989 & 12,913 & 133,275 & 109 \\
1990 & 13,421 & 153,796 & 143 \\
1991 & 13,804 & 170,601 & 178 \\
1992 & 13,673 & 188,780 & 198 \\
1993 & 13,938 & 189,700 & 184 \\
1994 & 14,799 & 211,914 & 225 \\
Total & 123,993 & $1,487,922$ & 1,402 \\
\hline
\end{tabular}

citations can contribute to the codification of knowledge claims into concept symbols.

The aim of the present study is to propose and test a criterion based on citations received and patterns of citations by a set of research papers over at least 25 years, to identify those which have stood the test of time and have a potential to continue further for the next 10 or more years. We term such research papers as long-lasting (LL). For this purpose, a longitudinal study of citations is conducted, of the papers authored by at least one researcher from India for the period 1985-1994 (10 years) and citations received till 2019. The criterion evolved in this study is a general one and may be applicable, with minor modifications, to any set of research papers as well as for measuring research contributions by an individual researcher as well as by any institution or from any country.

The objectives of this study are:

- To suggest a citation-based criterion for identification of L-L research papers from the test dataset of papers published between 1985 and 1994, employing citation records till 2019.

- To identify the disciplines which have contributed to L-L research papers reported from India between 1985 and 1994.

- To answer the following questions: (i) Are all the papers published in high impact journals L-L? (ii) Are all the papers receiving large citations L-L?

A citation-based method is developed for identifying potentially L-L papers, from all the papers listed in Scopus with at least one author from India. We report a test run using the database of research papers for 10 years (published between 1985 and 1994). Note the following. (i) The dataset is large enough, and comes from a variety of institutions, incorporating citation data for 25-35 years. (ii) After developing such a criterion, a subjectwise classification of L-L papers published from India is carried out and reported. This method is general and can be applied to any dataset of publications and citations. It can also be readily automated.
In the first part of the study, we suggest a method for analysing the entire dataset. Table 1 provides a brief summary of the entire dataset used. It includes citation data of over 123,993 research papers authored by at least one researcher from India. It may be seen that the average number of citations, for the period 1985-2019, for a paper published between 1985 and 1994 is approximately 12. This translates to about 0.25 citations per year, on an average, for a paper published in any standard refereed journal. This just reconfirms a well-known fact that most of the published work receives scanty citations.

Since we are interested in identifying potentially L-L papers based on longitudinal citation records, we need not look at papers which have received very low number of citations. We choose a cut-off value of 100 citations received by a paper from the year of publication to 2019 . The reason for choosing this value is that our study is based on citation records for 25-35 years, and a cut-off value of 100 would mean that the average number of citations per year lies between 3 and 4. For a smaller cut-off, e.g. 50 , the number of citations after 20 years of many papers would be just 0 or 1 , rendering the judgement of long-lived nature difficult. On the other hand, using a larger cut-off value of total number of citations, say 500 or 300 , will shortlist very few papers (55 or 143 respectively, from the entire dataset of 123, 993 research papers) for L-L analysis, missing out a large number of impact-making papers. In view of this, our chosen cut-off value of 100 for the total number of citations appears to be optimum. Incidentally, the cut-off value of 100 citations is a historical one. One may recollect that in the 1970 s Garfield ${ }^{22}$ had termed such papers as 'citation classics'.

It may be pointed out here that this cut-off value may be discipline-dependent and an appropriate, somewhat lower cut-off value may be chosen for disciplines such as mathematics, social sciences, etc. However, for most of the subject areas covered by Scopus, this cut-off value seems to be appropriate. It can be seen from Table 1 that the number of papers receiving over 100 citations is only 1,402. However, this set of 1,402 papers includes 35 


\section{RESEARCH COMMUNICATIONS}

Table 2. Study protocol employing long-term and short-term citation patterns for identifying long-lasting (L-L) original papers. See text and Tables 1,3 and 5 for details

Step 1: Data selection

Collect papers published between the years YS and YE, with at least one author from India as listed in Scopus. Record the citations from the year of publication (YP) to 2019.

Year of start $=1985$; Year of end $=1994$ (example years)

Step 2: Data set limitation

Filter out papers with $<100$ citations and also review articles. The remaining articles are shortlisted and taken up for further analysis.

Step 3: Citation analysis based on quarters

Divide the time interval between YP and 2019 in four approximately quarters. For a given paper in the shortlist, calculate the sum of citations $\left(N_{4}\right)$ for the years in the latest quarter, the total number of citations from YP to 2019 being $N$. Compute $R_{4}=N_{4} / N$. Select articles with $R_{4}$ value of 0.20 , with at least four average citations per year for the latest quarter. (Example year: YP $=1987$; latest quarter: 2012-2019.)

Step 4: Analysis of citation patterns during the latest 15 years (2005-2019)

Calculate the number of citations between the three 5-yr intervals: 2005-2009, 2010-2014 and 2015-2019. Omit those receiving less than 20 citations between 2015 and 2019 (five years). Also calculate the estimated number of citations (for 2020-2024) based on the extrapolation of the number of actual citations in the above-mentioned three intervals. Omit papers with less than 20 estimated citations from the shortlist. Thus the final list of potentially L-L papers is generated.

Step 5: Classification of L-L papers yearwise and subject-wise

Compile and sort the finally short-listed L-L papers yearwise and subject-wise (according to the broad subject list given by Scopus).

review articles which are not included in the subsequent analysis of L-L papers.

We have developed a citation-based step-by-step protocol for identifying the L-L papers (Table 2). We apply this protocol to the chosen dataset of 1367 shortlisted research papers and present the results of our analysis.

It may be noted that the protocol does not make use of the number of 'gaps' in the citations received by a research paper. The logic of elimination of papers in steps 3 and 4 is explained below.

1. Step 3: A value of $R_{4}=0.25$ would imply that the paper is receiving steady citations in the recent quarter. In order that potentially L-L papers are not missed out, we choose a typical $R_{4}$ with a cut-off value of 0.20 . We further ensure that at least four average citations per year are received for the latest quarter. The rest of the articles are eliminated.

2. Step 4: Papers with less than 20 citations in the most recent five years (i.e. average of 4 citations per year). Further, for the papers receiving 30 or less citations between 2015 and 2019, an extrapolation is carried out based on the number of citations received in the three slots of five years, viz. 2015-2019, 2010-2014 and 2005-2009. The papers for which this extrapolated value falls below 20 are also eliminated.

3. It is of interest to carry out subject-wise analysis of the L-L papers. These papers are grouped according to the subject classification given in Scopus. Such an analysis is expected to bring out the strength of research in various disciplines in the given database of publications.

4. In the last part of our work, we answer the following queries: Is every paper published in a high-impact journal L-L? What percentage the L-L papers is published in high-impact journals?

Figure 1 shows the typical patterns of citations received by six research papers from our chosen dataset (papers published between 1985 and 1994, receiving $\geq 100$ citations). The chosen paper is shown in a colour code given at the bottom of the curve. The $X$-axis shows the year of citation and the corresponding value on the $Y$-axis shows the number of citations received by that paper in that particular year. We briefly discuss these case studies below.

I. Papers not belonging to L-L category

(i) Paper shown in black colour (published in 1986): This exhibits a typical pattern of citations. It shows an overall steady growth with some ups and downs in citations, reaching over 10 citations after 15 years. After this, the number of citations shows an overall decline even after 20 years ( 2 or 3 citations per year).

(ii) Paper shown in maroon colour (published in 1987): In the first two years, it shows high citations, 31 and 39 respectively, followed by a sharp decline to 9 in the next year and has never received more than 4 citations afterwards. This paper clearly belongs to 'flash-in-the-pan' type.

(iii) Paper shown in grey colour (published in 1988): This exhibits a general steady growth in the first 20 years and a decline thereafter, with less than 5 average citations per year in the last five years.

II. Papers belonging to L-L category

(i) Paper shown in orange colour (published in 1989): This exhibits a general steady increase in citations for the first few years, with maximum citations (27) after 15 years followed by around 20 citations per year up to 2019 .

(ii) Paper shown in blue colour (published in 1990): The depicts the case of 'sleeping beauty', with 0 or very few citations in the first 13 years, reaching a first peak of 26 citations after 17 years. After this point, it shows large peaks, with citations in the range 50-60 per year continuing till 2019. 


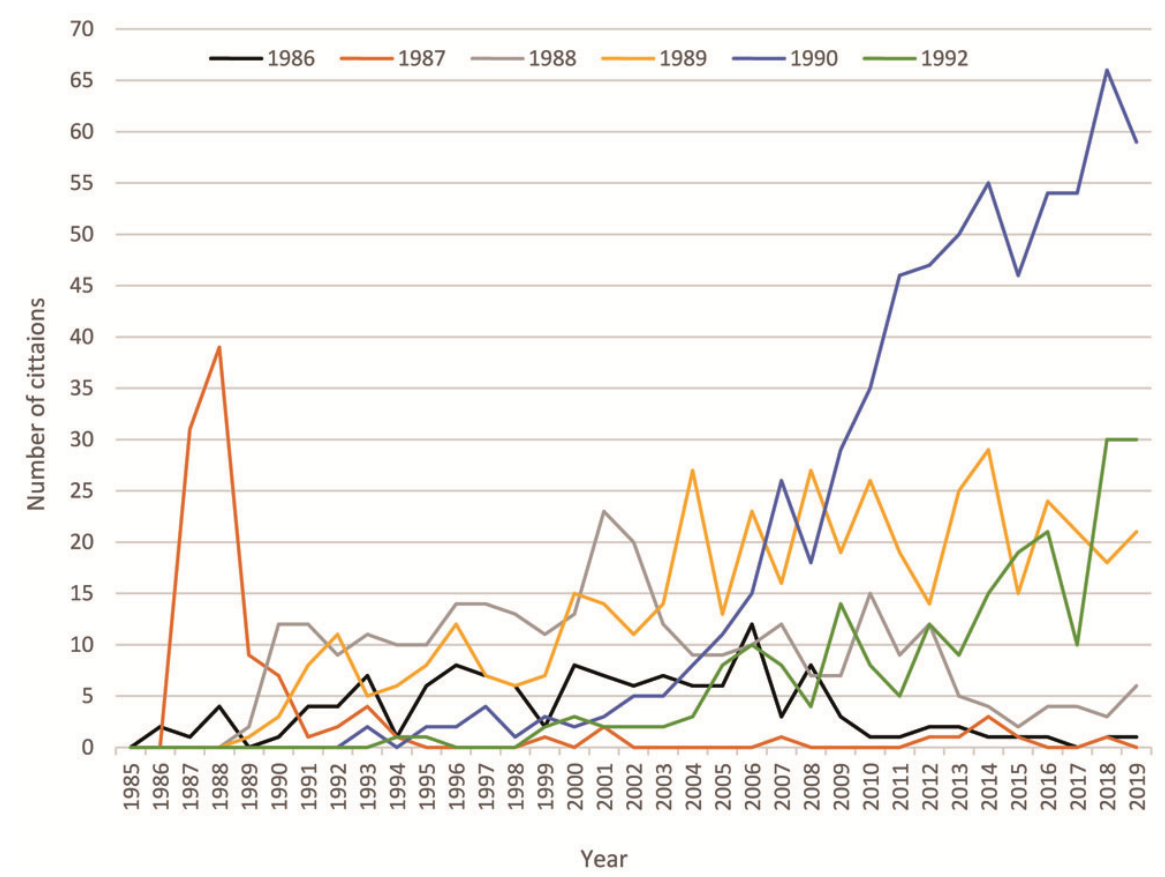

Figure 1. Illustration of the pattern of citations for some research articles (published between 1985 and 1994, colour-coded year of publication given in the bottom) studied. The $Y$-axis denotes number of citations received by a paper in the years shown on the $X$-axis. See text for details.

Table 3. Yearwise number of L-L papers published from India between 1985 and 1994 (10 years) identified by our protocol in Table 2. See text for details

\begin{tabular}{lccccccccccc}
\hline Year of publication & 1985 & 1986 & 1987 & 1988 & 1989 & 1990 & 1991 & 1992 & 1993 & 1994 & Total \\
\hline No. of L-L papers & 31 & 43 & 42 & 53 & 51 & 70 & 85 & 86 & 96 & 119 & 676 \\
\hline
\end{tabular}

(iii) Paper shown in light green colour (published in 1992): The curve is similar in nature to that in (i), showing very scanty citations till 2005, touching 10 citations only after 13 years. Thereafter it shows generally larger number of citations, ranging between 10 and 30 per year: a candidate for L-L category.

These qualitative considerations are clearly borne out by the application of our proposed criterion for L-L research papers. The cases in I above satisfy the criterion of an L$\mathrm{L}$ paper described in our protocol (Table 2) and those in II do not.

Applying the protocol in Table 2, we have scrutinized all the 1367 non-review-type articles in the dataset of 1402 original research articles, which have received 100 or more citations. Table 3 displays the results, showing a total of $676 \mathrm{~L}-\mathrm{L}$ papers. The number of L-L papers shows a steady increase from 1985 (31 papers) to 1994 (119 papers), as may be intuitively expected. The number of papers meeting the shortlisting criterion, viz. 100 or more total citations also shows an increase in this period (85 in 1985 to 225 in 1994). The papers published in 1985 have been receiving citations for 35 years, and the $31 \mathrm{~L}-\mathrm{L}$ papers identified by us in 2020 have the potential to con- tinue receiving citations for another 10 or more years. However, those published in 1994 have been in existence only for 25 years, and some of the 119 L-L papers identified by us may show a decay in the number of citations in another 5-10 years. It will be interesting to do the next round of citation analysis in 2025 .

Thus, we have a criterion for identifying L-L papers from a given database of papers published in a given time period and their long-term citations. Note that we have used citation data up to 2019 , i.e. for a rather long period (a minimum of 25 years and a maximum of 35 years) for this purpose. It would be worthwhile to get some further insights from the results of this analysis. Next we present subject-wise analysis of the L-L papers identified by us. The selected L-L papers published between 1985 and 1994 fall into 20 subject areas according to Scopus (Table 4). Such an analysis is helpful to identify the fields in which significant L-L contributions to science from India have been made on a global scale. A large number of L-L contributions are in Chemistry, Physics (including Astronomy), Medicine, Engineering (including Chemical Engineering), Agricultural and Biological Sciences, including Biochemistry. Computer Science, Materials Science, Environmental Science, Business Management and Mathematics follow. Social Sciences 
RESEARCH COMMUNICATIONS

Table 4. Subject-wise number of L-L papers published from India between 1985 and 1994 (10 years) using Scopus. See text for details

\begin{tabular}{|c|c|c|c|c|c|c|c|c|c|c|c|}
\hline Subject & 1985 & 1986 & 1987 & 1988 & 1989 & 1990 & 1991 & 1992 & 1993 & 1994 & Total \\
\hline Agricultural and Biological Sciences & 1 & 1 & 4 & 3 & 3 & 9 & 8 & 5 & 4 & 12 & 50 \\
\hline Biochemistry & 2 & 1 & 1 & 4 & 2 & 5 & 2 & 12 & 13 & 9 & 51 \\
\hline Business, Management and Accounting & 0 & 2 & 1 & 0 & 2 & 2 & 1 & 0 & 4 & 2 & 14 \\
\hline Chemical Engineering & 0 & 0 & 1 & 0 & 0 & 1 & 1 & 0 & 3 & 1 & 7 \\
\hline Chemistry & 6 & 8 & 5 & 5 & 9 & 8 & 18 & 7 & 14 & 18 & 98 \\
\hline Computer Science & 1 & 0 & 2 & 1 & 2 & 1 & 4 & 5 & 3 & 10 & 29 \\
\hline Earth and Planetary Sciences & 3 & 4 & 6 & 2 & 5 & 5 & 4 & 8 & 6 & 4 & 47 \\
\hline Economics & 1 & 0 & 0 & 0 & 0 & 1 & 1 & 1 & 2 & 2 & 8 \\
\hline Energy & 0 & 1 & 1 & 2 & 0 & 1 & 1 & 0 & 1 & 2 & 9 \\
\hline Engineering & 5 & 2 & 1 & 9 & 4 & 5 & 7 & 13 & 8 & 19 & 73 \\
\hline Environmental Science & 3 & 3 & 0 & 6 & 1 & 3 & 1 & 4 & 8 & 2 & 31 \\
\hline Immunology and Microbiology & 1 & 0 & 0 & 0 & 0 & 0 & 1 & 0 & 0 & 1 & 3 \\
\hline Materials Science & 0 & 4 & 4 & 4 & 0 & 4 & 1 & 5 & 3 & 1 & 26 \\
\hline Mathematics & 0 & 1 & 2 & 4 & 2 & 1 & 3 & 1 & 1 & 2 & 17 \\
\hline Medicine & 5 & 9 & 6 & 2 & 10 & 9 & 15 & 11 & 6 & 15 & 88 \\
\hline Multidisciplinary & 0 & 2 & 0 & 1 & 2 & 3 & 4 & 2 & 4 & 1 & 19 \\
\hline Neuroscience & 0 & 0 & 0 & 0 & 0 & 0 & 0 & 1 & 0 & 0 & 1 \\
\hline Physics and Astronomy & 3 & 4 & 7 & 11 & 4 & 11 & 14 & 11 & 15 & 17 & 97 \\
\hline Psychology & 0 & 0 & 0 & 0 & 0 & 0 & 0 & 0 & 1 & 0 & 1 \\
\hline Social Science & 0 & 1 & 1 & 0 & 2 & 1 & 1 & 0 & 0 & 1 & 7 \\
\hline Total & 31 & 43 & 42 & 54 & 48 & 70 & 87 & 86 & 96 & 119 & 676 \\
\hline
\end{tabular}

Table 5. Number of L-L research papers published in 1992, 1993 and 1994 identified by our protocol. The papers published in journals with impact factor (IF) greater than 5 are also listed. See text for details

\begin{tabular}{lcc}
\hline Year & $\begin{array}{c}\text { No. of L-L } \\
\text { papers }\end{array}$ & $\begin{array}{c}\text { No. of L-L papers in journals } \\
\text { with IF }>5\end{array}$ \\
\hline 1992 & 86 & 28 \\
1993 & 96 & 26 \\
1994 & 119 & 27 \\
\hline
\end{tabular}

Table 6. Number of L-L research papers published in 1992, 1993 and 1994 receiving more than 150 citations till 2019 along with those identified as L-L by our protocol

\begin{tabular}{lcc}
\hline Year & $\begin{array}{c}\text { No. of papers with } \\
>150 \text { citations }\end{array}$ & $\begin{array}{c}\text { No. of L-L papers } \\
\text { among them }\end{array}$ \\
\hline 1992 & 82 & 58 \\
1993 & 83 & 61 \\
1994 & 88 & 68 \\
\hline
\end{tabular}

and Humanities are the fields that do not show major L-L contributions. As noted above, some of the results may be partly responsible for the cut-off criterion used in our protocol and need to be revisited.

Publishing in high IF journals is often overemphasized, though not all articles published in such journals are well-cited. We analyse the number of L-L research papers published in 1992, 1993 and 1994 and check the high impact of journals. Table 5 shows that only about $25-30 \%$ of these L-L papers are published in high-impact journals, the remaining are published in journals with IF less than 5 . The choice of 5 as the cut-off value for high-impact journals is somewhat subjective. We may choose a subject-dependent cut-off value for identifying high-impact journals in that subject.

It is noticed that many L-L papers are published in journals with $\mathrm{IF}<2$. This highlights that there is no clear relationship between journal IF and L-L papers published therein.

Are all well-cited papers L-L? We have explored the answer to this question by taking the test data of citations for papers published in 1992, 1993 and 1994, which have received 150 or more citations up to 2019 (Table 6). About 25\% papers from this set do not get sustained citations after a period of 20 years and do not belong to the L-L category.

It will be indeed interesting to revisit the citation database chosen in this study after a period of five years (2025) and check whether the L-L papers identified by citations up to 2019 continue to be so using the citation database up to 2024. Many of the papers in our database would have reached a ripe age of at least about 40 years in 2024.

Our protocol (Table 2) for identifying L-L research papers was developed after a detailed study of the citation patterns, a few typical examples of which were displayed in Figure 1. In the first step, we shortlist papers which have received 100 or more citations. The $R_{4}$ parameter checks the ratio of citations received by a paper during the last quarter of its life till 2019, to the total ones since its publication. We have found that a cut-off value of 0.20 is able to filter-off papers which do not show the potential to be of L-L type.

The recent trends in citations (during the last five years (2015-19) out of the total of 15 years) are examined after 
this stage. Papers with less than 20 actual or extrapolated citations (in agreement with the suggested value of 4 average citations per year) are omitted. Overall, we have a robust protocol for identifying long-lasting research papers.

Using this protocol, we have explored citation patterns of over 1,23,993 original research papers in Scopus published from India between 1985 and 1994. Our longitudinal study resulted into 676 (about $0.5 \%$ of the total) potentially L-L papers. Some main observations from our study are listed below.

- We found that some of the papers in high IF journals are well-cited, but some are not ${ }^{23}$.

- Several examples of 'sleeping beauties"11 as well as 'flash-in-the-pan'12 behaviour of citations were noticed.

- We found that the suggestion to employ the journal IF and the number of early citations as predictors of long-term citations may not be of general use ${ }^{18,19,24}$. Further, the model of Xiao et al. ${ }^{20}$ on predictions based on short-term citations for long-term ones also has limitations.

- On the other hand, we found that the statement of Leydesdorff et $a l^{21}$ that 'a short-term citation is like a citation currency, whereas the long-term citation may contribute to the codification of knowledge' indeed provides the gist of the citation scenario.

- Our study has also found that some highly cited works get cited even after 25 years, as suggested by Walters ${ }^{13}$. However, there are some exceptions to this trend.

An index based on L-L papers would be useful for various academic purposes such as for granting recognitions, research projects and awards, for individual senior researchers.

An appraisal of institutions, based on the norms of research quality could include a parameter such as L-L papers from the faculty. This will be more meaningful than an analysis based on the number of publications, their citations and IFs of the relevant journals.

After developing a working protocol for the identification of L-L research papers, we plan to fine-tune the same and automate its implementation. Future studies, we will make our protocol more robust, and test it on more diverse and larger datasets of published research papers.

1. Garfield, E., Science citation index - a new dimension in indexing. Science, 1964, 144, 649-654.

2. Roldan-Valadez, E., Salazar-Ruiz, S. Y., Ibarra-Contreras, R. and Rios, C., Current concepts on bibliometrics: a brief review about impact factor, eigenfactor score, CiteScore, SCImago Journal Rank, source-normalised impact per paper, $h$-index, and alternative metrics. Irish. J. Med. Sci., 2019, 188, 939-951.

3. Seglen, P. O., Why the impact factor of journals should not be used for evaluating research. BMJ, 1997, 314, 497.
4. Falagas, M. E., Pitsouni, E. I., Malietzis, G. A. and Pappas, G., Comparison of PubMed, Scopus, Web of Science, and Google Scholar: strengths and weaknesses. FASEB J., 2008, 22, 338-342.

5. Wouters, P. et al., Rethinking impact factors: better ways to judge a journal. Nature, 2019, 569, 621-623.

6. Garfield, E., Premature discovery or delayed recognition - Why. Curr. Cont., 1980, 21, 5-10.

7. Aversa, E., Citation patterns of highly cited papers and their relationship to literature aging: a study of the working literature. Scientometrics, 1985, 7, 383-389.

8. Glänzel, W. and Schoepflin, U., A bibliometric study on ageing and reception processes of scientific literature. J. Inf. Sci., 1995, 21, 37-53.

9. Moed, H. F., Leeuwen, T. N. V. and Reedijk, J., A new classification system to describe the ageing of scientific journals and their impact factors. J. Doc., 1998, 54, 387-419.

10. Aksnes, D. W., Characteristics of highly cited papers. Res. Eval., 2003, 12, 159-170.

11. van Raan, A. F. J., Sleeping beauties in science. Scientometrics, 2004, 59, 467-472.

12. van Daylan, H. P. and Henkens, K. N., Signals in science - on the importance of signaling in gaining attention in science. Scientometrics, 2005, 64, 209-233.

13. Walters, G. D., The citation life cycle of articles published in 13 American Psychological Association journals: a 25-year longitudinal analysis. J. Am. Soc. Inf. Sci. Technol., 2011, 62, 16291636.

14. Wang, D., Song, C. and Barabási, A. L., Quantifying long-term scientific impact. Science, 2013, 342, 127-132.

15. Abt, H. A., Long-term citation histories of astronomical papers. Astron. Soc. Pac., 1981, 93, 207.

16. Nash, J. R., Araújo, R. J. and Shideler, G. S., Contributing factors to long-term citation count in marine and freshwater biology articles. Learn. Publ., 2018, 31, 131-139.

17. Kosteas, V. D., Predicting long-run citation counts for articles in top economics journals. Scientometrics, 2018, 115, 13951412.

18. Stegehuis, C., Litvak, N. and Waltman, L., Predicting the longterm citation impact of recent publications. J. Informetr., 2015, 9, $642-657$.

19. Abramo, G., D’Angelo, C. A. and Felici, G., Predicting publication long-term impact through a combination of early citations and journal impact factor. J. Informetr., 2019, 13, 32-49.

20. Xiao, S. et al., On modeling and predicting individual paper citation count over time. In Proceedings of the Twenty-Fifth International Joint Conference on Artificial Intelligence (IJCAI-16), 2016, pp. 2676-2682.

21. Leydesdorff, L., Bornmann, L., Comins, J. A. and Milojević, S., Citations: Indicators of quality? The impact fallacy. Front. Res. Metr. Anal., 2016, 1, 1.

22. Garfield, E., Introducing citation classics - human side of scientific reports. Curr. Cont., 1977, 1, 5-7.

23. Garfield, E., How to use citation analysis for faculty evaluation and when is it relevant? Part 1. Curr. Cont., 1983, 44, 5-13.

24. Costas, R., van Leeuwen, T. N. and van Raan, A. F., Is scientific literature subject to a 'sell-by-date'? A general methodology to analyze the durability of scientific documents. J. Am. Soc. Inf. Sci. Technol., 2010, 61, 329-339.

Received 1 November 2020; accepted 4 November 2020

doi: $10.18520 / \mathrm{cs} / \mathrm{v} 120 / \mathrm{i} 1 / 209-214$ 